\title{
Abstract
}

\section{Dark Matter, Dark Energy and Something Else in 5D Theory ${ }^{\dagger}$}

Boris G. Aliyev

check for

updates

Citation: Aliyev, B.G. Dark Matter, Dark Energy and Something Else in 5D Theory . Phys. Sci. Forum 2021, 2, 7. https://doi.org/10.3390/ ECU2021-09278

Academic Editor: Lorenzo Iorio

Published: 22 February 2021

Publisher's Note: MDPI stays neutral with regard to jurisdictional claims in published maps and institutional affiliations.

Copyright: (C) 2021 by the author. Licensee MDPI, Basel, Switzerland. This article is an open access article distributed under the terms and conditions of the Creative Commons Attribution (CC BY) license (https:/ / creativecommons.org/licenses/by/ $4.0 /)$.
Architecture Department, V.I. Surickov Moscow State Art Institute, Altendorfer Str. 28, 09113 Chemnitz, Germany; bgaliyev@mail.ru

+ Presented at the 1st Electronic Conference on Universe, 22-28 February 2021; Available online: https://ecu2021.sciforum.net/.

Abstract: It is shown, that $(4+1)$-and $(3+1+1)$-reductions of the geodetic equations in 5D theory with scalar field $\varphi=\sqrt{-G_{55}}$ and out of the 5D optics inevitably lead to the new concept of the Lorentz-type relativistic mass $\hat{m}_{0}$ of the $5 \mathrm{D}$ test particle. Due to the imposition of the $x^{5}$-cylindricity condition, one can obtain an integral of the 5D particle's motion along $x^{5}$; it gives its electric charge. Thus, one can obtain an exact expression for $\hat{m}_{0}$. In this expression $\hat{m}_{0}$ depends on the field $\varphi$ through an electric charge, so there is not probably any need for a scalar charge in Nature at all. Furthermore, one can compactly express the $\hat{m}_{0}$ through the mass angle $\chi_{n}$ and additionally hypothesize about the possible complex structure of $\hat{m}_{0}$. It soundly leads one to the deeper understanding of the quantum properties of the matter. All of these conceptions also turn one to the idea that this mass $\hat{m}_{0}$ may contribute to the recently discovered in the Universe dark matter and dark energy and also be one of the possible reasons for the Universe's expansion. The next suggestive result in the 5D theory is connected with the $(4+1)$-reduction of the 5D Ricci identities. It leads one to certain connections between the $4 \mathrm{D}$ physicogeometrical values and permits one to obtain the first pair of Maxwell equations with the non-zero soliton-type r.h.s. and establishes the connection with the second pair of them. It subsequently leads one to the idea about the magnetic monopole's existence in the early Universe. It is shown, that this non-zero r.h.s. vanishes together with the imposition of the $x^{5}$-cylindricity condition. The peculiarity of this process permits one to hypothesize soundly about the existence of the topological second-order transition in the Universe, which leads one to the superfluid state and possibly accelerates its expansion.

Keywords: 5D relativistic mass concept; scalar gravitational field; 5D geodetic equations; cylindrical symmetry condition; 5D Ricci identities; Maxwell equations; magnetic monopole; topological secondorder phase transition in cosmology

Supplementary Materials: The following are available online at https:/ / www.mdpi.com/article/10 .3390/ECU2021-09278/s1.

Institutional Review Board Statement: Not applicable.

Informed Consent Statement: Not applicable. 